\section{Tricks of the Trade: Particle Mounting Methods}

Walter C. McCrone, McCrone Research Institute

Those of us who have particle identification problems sometimes have very small samples, say, only a few nanograms - even less sometimes. We also like to make up permanent Aroclor 5442 preparations of these tiny samples for safe keeping and future reference. If we mount such small samples in a normal drop of Aroclor filling an $18 \mathrm{~mm}$ diameter coverslip, we may spend a few frustrating minutes looking for the sample in a few acres of Aroclor.

My solution is to use a very tiny Aroclor droplet under a small coverslip. Ten millimeter or smaller round coverslips are available from Erie Scientific in Portsmouth, New Hampshire. To obtain a suitable Aroclor droplet (Figure 1), I keep my Aroclor-filled canada balsam bottle nearly empty $\left(2-3 \mathrm{~mm}\right.$ deep) on my hotplate at $98^{\circ} \mathrm{C}$ and with a fine-tipped glass rod permanently therein. I place my clean microscope slide with a centrally Sharpie pen-marked " $q$ " on the under surface on the hotplate. Ten seconds later, I remove the glass rod and tick the tip wet with Aroclor to the circle of " $p$ " on the other side of the slide. I then place a clean $10 \mathrm{~cm}$ round coverslip on this drop (no sample), heat it to $100^{\circ} \mathrm{C}$, and press on the coverslip to thin the Aroclor. It then cools to room temperature as I walk to the refrigerator with the prep and a razor blade. After 10 seconds for the slide on the cold freezer compartment surface, I remove the slide and flick off the coverslip with the razor under one edge. Now, back to the stereo microscope where I use a No. 2 or 3 tungsten needle to place the sample at the center of the Aroclor circle and replace the coverslip (Figure 2). Now, another 10 seconds on the hotplate followed by removal to a heat insulating Post-It pad and quick use of a coverslip gripper-particle crusher and disperser (i.e., a pencil eraser). I disperse the sample into single

\section{ANALOG/DIGITAL PRINTERS}

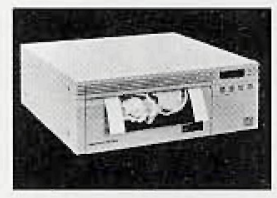

Seikosha Video Printers

- 256 Grey Levels

- $10 \mathrm{MHz}-135 \mathrm{MHz}$

- 5e-19c-40c Hardcopies

- $3 \times 4,81 / 2 \times 8$ or

$81 / 2 \times 11$ Formats

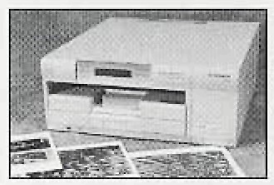

Mitsubishi Printers

- Dye-Sublimation Printers

- Analog/Digital Input

- 16.7 Million Colors

- $4 \times 4$ thru $12 \times 18$ Formal

- Thermal Greyscale P'rinters

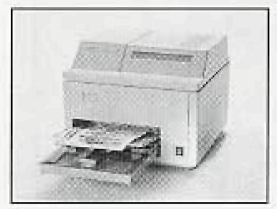

Tektronix Printers

- Dye-Sublimation Printers

- Digital/Postscript Input

- 16.7 Million Colors

- $81 / 2 \times 11$ Thru $81 / 2 \times 14$

- $\mathrm{TCP} / \mathrm{IP}$ and Ethertalk

VITAL IMAGE TECHNOLOGY INC. OFFERS COMPLETE SALES AND SERVICE INCLUDING FREE INSTALLATION IN MOST PARTS OF THE UNITED STATES.
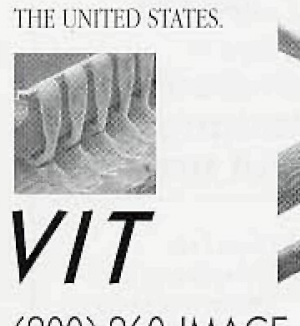

(800) 860-IMAGE

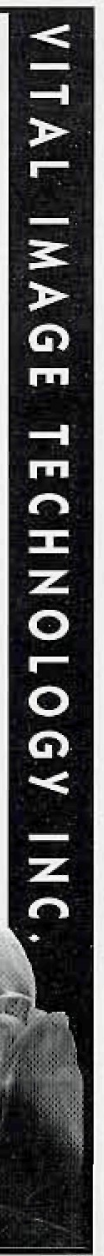

particles in a small area at the center of the Aroclor preparation. After circling its expanse on top of the coverslip (Figure 3) I am ready to find and examine the particle(s) by PLM.

Once in a great while, I then find I cannot identify one of the important components of this Aroclor preparation by size, shape, color, refractive indices, and birefringence. I must then get one of the biggest of those particles out, cleaned off and remounted in a different liquid or for SEM/EDS or FTIRI microscopy. This I do, starting with circling the chosen particle location on top of the coverslip. I then invert the slide and trace the particle circle on the bottom of the slide. Then back to the freezer to flick off the coverslip.

A fine-tipped tungsten needle, then draws a square through the Aroclor and around the particle. With the same needle working under a small drop of water over the particle on top of the Aroclor, I spear the square of Aroclor (and particle) and bring it out through the water drop surface to a clean slide. The Aroclor is removed using a drawn-out polyethylene pipette filled with amyl acetate leaving a clean particle. A small droplet placed near the particle is drawn by a nearly flat No. 2 needle tip over the particle, hesitating a few seconds for the Aroclor to dissolve and then continuing on to move the amhyl acetate droplet plus Aroclor away from the particle. Several airborne trips back to the particle and drawing more droplets away usually leaves a clean particle in less than 30 seconds total time. Needless to say, this operation takes place under the stereo at times 40-50.

The dry particle is then mounted in a different liquid for PLM, for SEM/EDS on a beryllium stub or in a diamond cell for FTIR/microscopy.

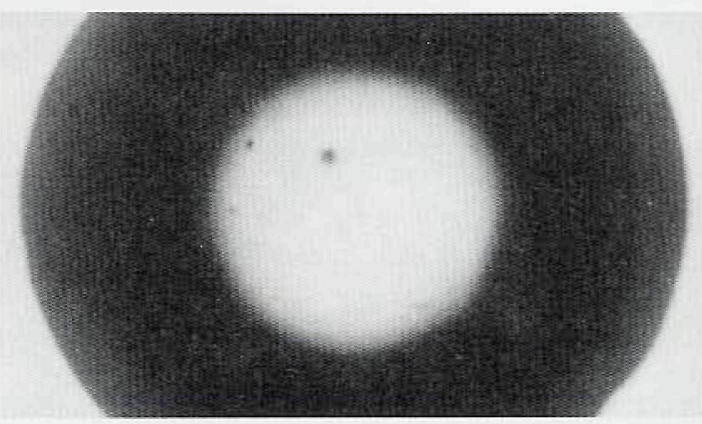

Figure 1: Droplet of $<2 \mathrm{~mm}$ Aroclor 5442 on the slide, $25 X$.

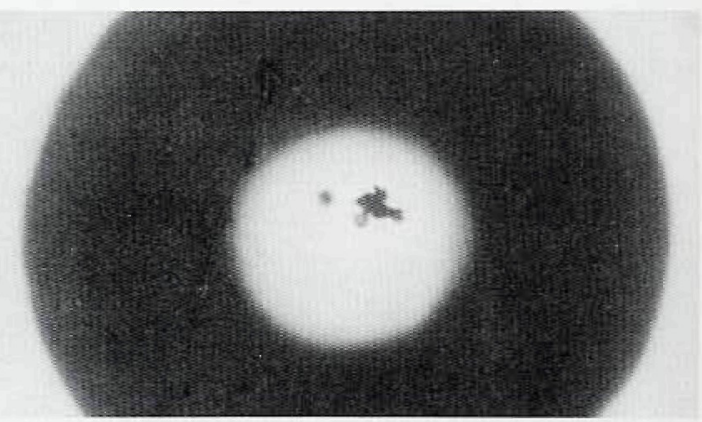

Figure 2: Aroclor droplet plus sample.

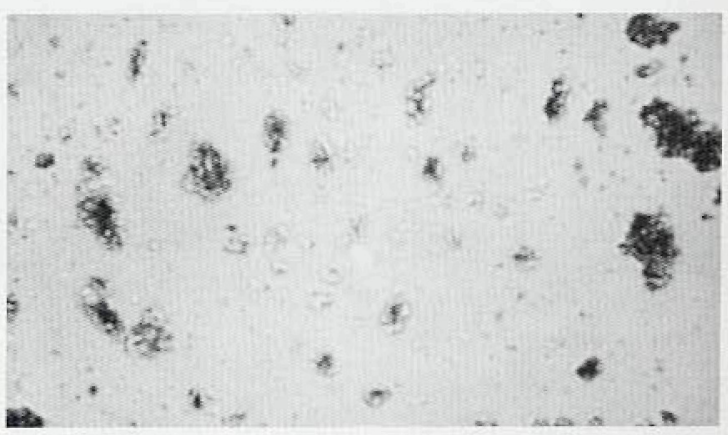

Figure 3: Dispersed particles of the sample in a $4 \mathrm{~mm}^{2}$ area.

Reprinted from Volume 44:1 10 (1996) of the MICROSCOPE from the McCrone Research Institute. 


\section{WHEN RESULTS COUNT APPLY TH THE TOTAL SOLUTION.}

When you need fast, accurate results for your microanalysis applications, count on NORAN Instruments' VOYAGER to deliver the right answer. We offer a full family of software that works together to solve the most challenging EDS, Digital Imaging, and WDS analytical problems. From Intelligent Qual automatic peak identification to our new WDS Automation and Analysis, VOYAGER has the total system solution.

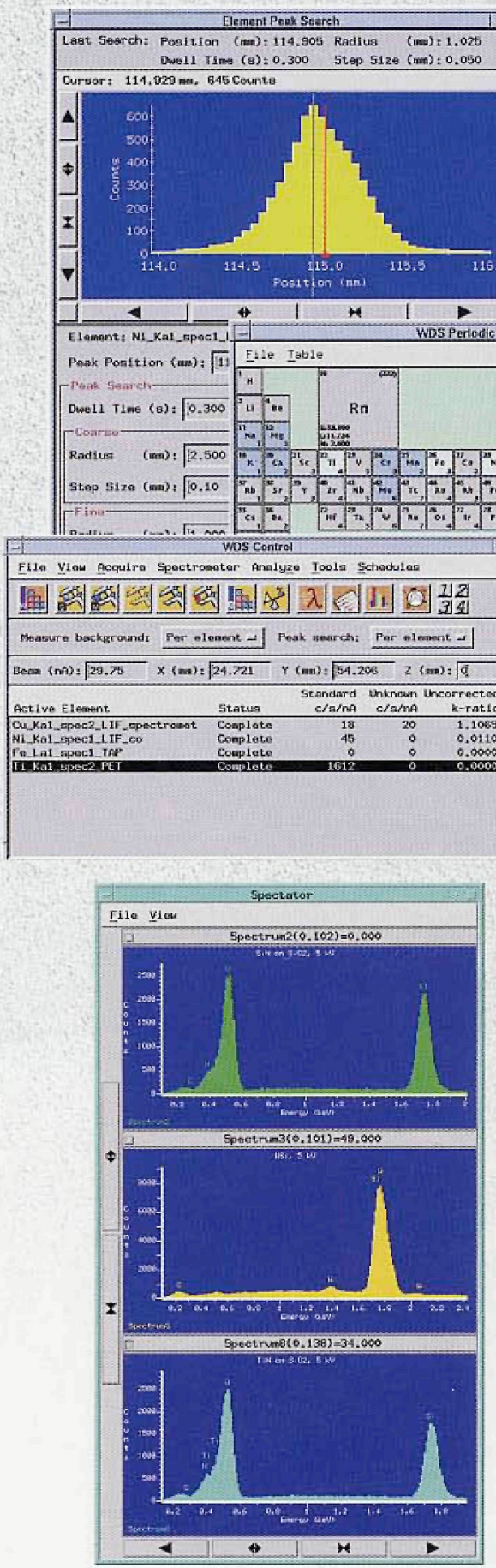

Our new WDS application for

VOYAGER is powerful, yet easy to use.

Our design lets you optimize the

analysis setup for single elements,

for the best results possible.
Powerful Analysis Manager software lets you automate your EDS, WDS, Imaging, Feature Sizing, and other laboratory procedures. With fully matrixcorrected quantitative maps, you can easily determine the distribution of element weight percentages in your sample.

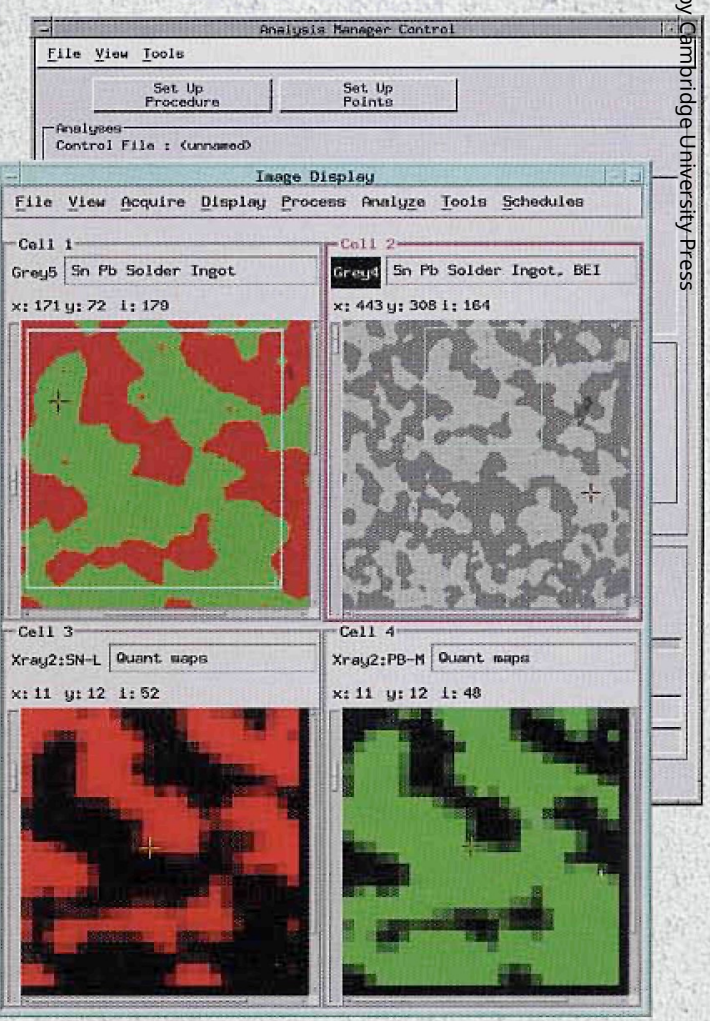

VOYAGER's Intelligent Qual quickly and accurately identifies spectral peaks, even in tough overlap situations such as Ti- $L$ with $N-K$, and $W-M$ with Si-K.

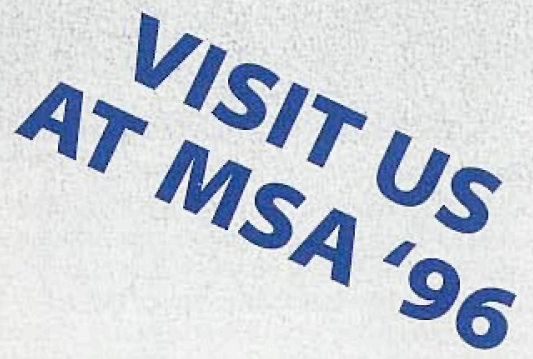

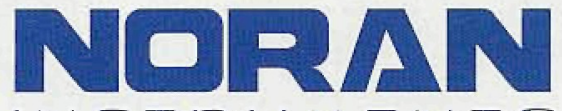

INSTRUMENTS

NORAN Instruments Inc., Corporate Headquarters,

2551 West Beltline Highway, Middleton, W1 53562-2697 USA Tel.: 6088316511 , Fax: 6088367224

Internet: voyager info@noran.com

World Wide Web: http://www.noran.com

NORAN Instruments B.V., Tel.: 3135 6998888, Fax: 31356949913 NORAN Instruments GmbH, Tel.: 49725197910 , Fax: 497251979111 NORAN Instruments Japan, Tel.: 8133298 8451, Fax: 81332988441 NORAN Instruments Korea, Tel.: 82342708 3091, Fax: 823427083093 NORAN Instruments U.K., Tel.: 441908696290 , Fax: 441908696292 